\title{
Induced encystment improves resistance to preservation and storage of Acanthamoeba castellanii
}

\author{
S. J. CAMPBELL ${ }^{1}$, P. R. INGRAM ${ }^{2}$, C. W. ROBERTS ${ }^{2}$ and F. L. HENRIQUEZ ${ }^{1 *}$ \\ ${ }^{1}$ School of Engineering and Science, University of the West of Scotland, Paisley PA1 2BE, Scotland \\ ${ }^{2}$ Strathclyde Institute of Pharmacy and Biomedical Sciences, University of Strathclyde, Glasgow G4 0NR, Scotland
}

(Received 9 Fune 2008; revised 4 Fuly and 4 August 2008; accepted 12 August 2008)

\section{S U M MAR Y}

Several conditions that allow the preservation, storage and rapid, efficient recovery of viable Acanthamoeba castellanii organisms were investigated. The viability of trophozoites (as determined by time to confluence) significantly declined over a period of 12 months when stored at $-70{ }^{\circ} \mathrm{C}$ using dimethyl sulfoxide (DMSO; 5 or $10 \%$ ) as cryopreservant. As A. castellanii are naturally capable of encystment, studies were undertaken to determine whether induced encystment might improve the viability of organisms under a number of storage conditions. A. castellanii cysts stored in the presence of $\mathrm{Mg}^{2+}$ at $4{ }^{\circ} \mathrm{C}$ remained viable over the study period, although time to confluence was increased from approximately 8 days to approximately 24 days over the 12 -month period. Storage of cysts at $-70{ }^{\circ} \mathrm{C}$ with DMSO $(5$ or $10 \%)$ or $40 \%$ glycerol, but not $80 \%$ glycerol as cryopreservants increased their viability over the 12 -month study period compared with those stored at room temperature. Continued presence of $\mathrm{Mg}^{2+}$ in medium during storage had no adverse effects and generally improved recovery of viable organisms. The present study demonstrates that $A$. castellanii can be stored as a non-multiplicative form inexpensively, without a need for cryopreservation, for at least 12 months, but viability is increased by storage at $-70{ }^{\circ} \mathrm{C}$.

Key words: Acanthamoeba, preservation, storage, cyst, trophozoite.

\section{INTRODUCTION}

Acanthamoeba species are normally free-living protozoa, their transmission and spread does not depend on a host and they are found almost ubiquitously in the environment (reviewed by Marciano-Cabral and Cabral, 2003). Several species are pathogenic, causing granulomatous amoebic encephalitis (GAE) and cutaneous acanthamoebiasis in immunocompromised or immunosuppressed hosts, and amoebic keratitis (AK) in immunocompetent individuals (reviewed by Marciano-Cabral and Cabral, 2003; Schuster and Visvesvara, 2004). As the importance of this pathogen is recognized there has been an increase in the number of studies, particularly those investigating new antimicrobials, that may improve treatment (Schuster and Visvesvara, 1998; Mattana et al. 2004; McBride et al. 2007; Henriquez et al. 2008).

The ability to preserve protozoa in a non-actively dividing state has a number of advantages. Firstly, it reduces the material and labour costs of continued culture and passage, secondly it reduces the chance of selection for culture-adapted organisms observed during continued tissue culture and it also allows the

* Corresponding author: School of Engineering and Science, University of the West of Scotland, Paisley PA1 2BE, Scotland. Tel: +01418483119. Fax: +01415484823. E-mail: fiona.henriquez@uws.ac.uk possibility to maintain more than one isolate in a single location. Furthermore, it may facilitate transport of isolates without regard to time. The cryopreservation of Acanthamoeba and other free-living amoebae has been previously described (Seo et al. 1992; John et al. 1994; John and John, 1996; Alejandre-Aguilar et al. 1998; Gonzalez-Robles et al . 2001). The slow cooling (overall $0.7^{\circ} \mathrm{C} / \mathrm{min}$ ) of freeliving amoeba trophozoites, Acanthamoeba culbertsoni, A. polyphaga, Naegleria fowleri and N. gruberi, with $7 \cdot 5 \%$ dimethylsulfoxide (DMSO) or $7 \cdot 5 \%$ glycerol produced 2-39\% viable trophozoites after 60 days in liquid nitrogen (Seo et al. 1992). John et al. (1994) reported that recovery of $A$. castellanii trophozoites decreases with longer storage times at $-70{ }^{\circ} \mathrm{C}$ with $12 \%$ DMSO, $20 \%$ heat-inactivated bovine calf serum and $4 \%$ glucose with the greatest reduction during the first year of storage (John and John, 1996). Alejandre-Aguilar et al. (1998) found that the viability of $A$. castellanii trophozoites increased when stored with $10 \%$ DMSO in liquid nitrogen if an equilibrium temperature of $4{ }^{\circ} \mathrm{C}$ was used.

As we found that our ability to recover viable trophozoites from cryopreservation reduced with time we sought an alternative method. Acanthamoeba exist as active trophozoites or dormant cysts. Encystment occurs when the organism is exposed to adverse environmental conditions such as absence of nutrients, biocides, fluctuations in temperature and 


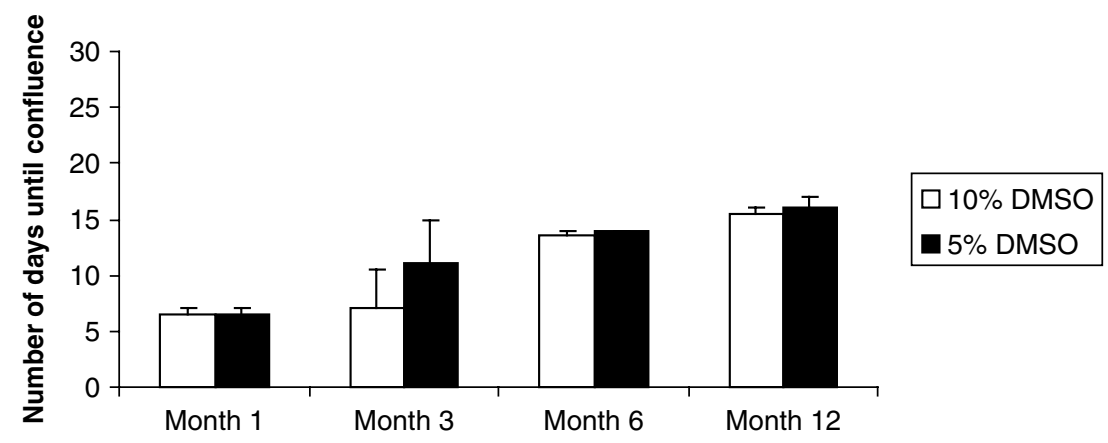

Fig. 1. The average time (days) until confluence for Acanthamoeba castellanii trophozoites stored in RM with $10 \%$ or $5 \%$ DMSO at $-70{ }^{\circ} \mathrm{C}$. The length of time to reach confluence increased the longer time spent in storage for all samples from $6 \cdot 5$ to 16 days. The data represent the mean of 2 replicate experiments.

pH (reviewed by Schuster, 2002). Encystment can be induced in the laboratory by the addition of $\mathrm{MgCl}_{2}$ to the medium (reviewed by Schuster, 2002). We hypothesized that encystment of Acanthamoeba may increase their viability during cryopreservation or eliminate the need for it completely.

Herein, a number of different storage conditions for A. castellanii trophozoites and cysts are compared. The effectiveness of each condition was determined via microscopical observations of the time required to reach confluence.

\section{MATERIALS AND METHODS}

\section{Maintenance of A. castellanii trophozoites}

Acanthamoeba castellanii (Neff strain) was kindly donated by Keith Vickerman (Glasgow, UK). Trophozoites were routinely grown in RM containing $2 \%$ mycological peptone (Sigma, Poole, UK) and $0.9 \%$ maltose (Sigma, Poole, UK), supplemented with $125 \mu \mathrm{g}$ penicillin/streptomycin and $2 \cdot 5 \mu \mathrm{g} / \mathrm{ml}$ amphotericin B (Sigma, Poole, UK) as previously described (McBride et al. 2005). They were incubated until confluent at room temperature in $75-\mathrm{cm}^{2}$ tissue-culture flasks, followed by either subculture or harvest by mechanical detachment.

\section{Preparation of cysts}

The RM was removed from confluent $75-\mathrm{cm}^{3}$ tissueculture flasks and replaced by $10 \mathrm{ml}$ of encystment medium (EM) (20 mM Tris- $\mathrm{HCl}, \mathrm{pH} 8 \cdot 8,100 \mathrm{~mm}$ $\mathrm{KCl}, 8 \mathrm{~mm} \mathrm{MgSO}_{4}, 0.4 \mathrm{~mm} \mathrm{CaCl}, 1 \mathrm{~mm} \mathrm{NaHCO}_{3}-$ all reagents from Sigma) (Neff et al. 1964) and monitored until cysts had formed (2-3 days). The cysts were then collected by centrifugation at $1200 \mathrm{~g}$ for 5 min resuspended in (EM) and counted using a haemocytometer. Cysts were adjusted to $3 \times 10^{5} / \mathrm{ml}$, added to microcentrifuge tubes (ThermoElectron, $\mathrm{UK}$ ) and centrifuged at $20000 \boldsymbol{g}$ for $5 \mathrm{~min}$. The EM was then removed and the cysts were resuspended in $1 \mathrm{ml}$ of the appropriate medium for storage: (1) EM or RM with (a) $10 \%$ DMSO, (b) $5 \%$ DMSO, (c) $2 \cdot 5 \%$

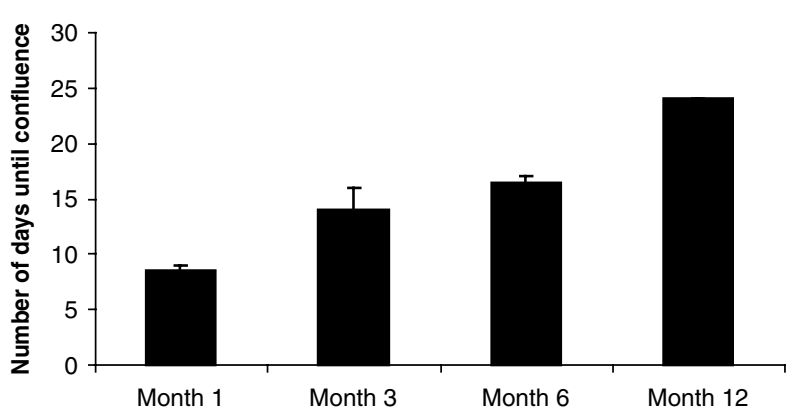

Fig. 2. The average time (days) until confluence for Acanthamoeba castellanii cysts stored in EM at $4{ }^{\circ} \mathrm{C}$. The length of time to reach confluence increased the longer time spent in storage from $8 \cdot 5$ to 24 days. The data represent the mean of 2 replicate experiments.

DMSO, (d) $80 \%$ glycerol, (e) $40 \%$ glycerol and (f) $20 \%$ glycerol or (2) EM alone. EM and RM suspensions with glycerol or DMSO were then transferred to a cryopreservation tube and stored at $-70{ }^{\circ} \mathrm{C}$ and $\mathrm{EM}$ alone was placed at $4{ }^{\circ} \mathrm{C}$ for $1,3,6$ and 12 months.

\section{Preparation of trophozoites}

Trophozoites were collected from newly confluent cultures by mechanical disruption followed by centrifugation at $1200 \boldsymbol{g}$ for $5 \mathrm{~min}$ and resuspended in RM. Then $3 \times 10^{5}$ trophozoites in $1 \mathrm{ml}$ of suspension were added to microcentrifuge tubes and centrifuged at $1200 \mathrm{~g}$ for $5 \mathrm{~min}$. The RM was then removed and the trophozoites were resuspended in RM, counted using a haemocytometer and adjusted to $3 \times 10^{5} / \mathrm{ml}$ in the appropriate medium for storage: RM with (a) $10 \%$ DMSO, (b) $5 \%$ DMSO, (c) $2 \cdot 5 \%$ DMSO, (d) $80 \%$ glycerol, (e) $40 \%$ glycerol and (f) $20 \%$ glycerol. The trophozoite suspensions were then transferred to a cryopreservation tube (Nunc, Denmark) and stored at $-70{ }^{\circ} \mathrm{C}$ for $1,3,6$ and 12 months.

\section{Recovery of Acanthamoeba}

After the appropriate length of time in storage (1, 3, 6 and 12 months) each cryovial was thawed slowly at 

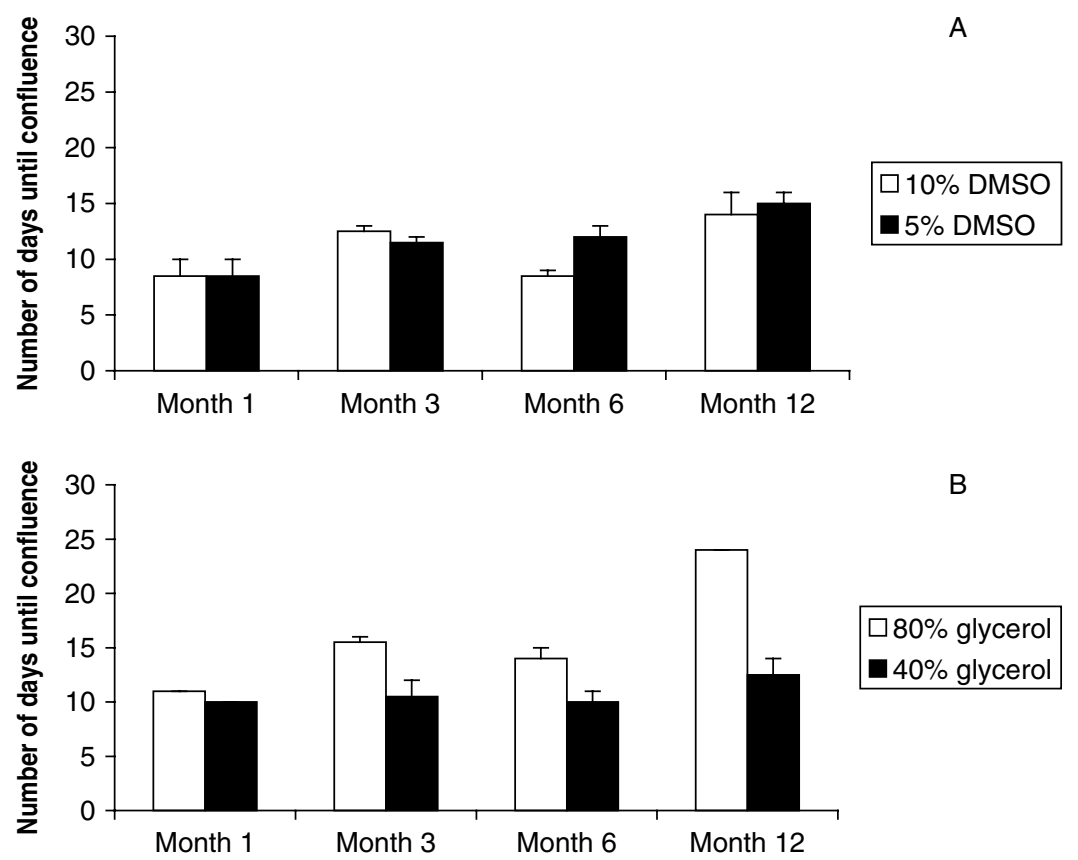

Fig. 3. The average time (days) until confluence for Acanthamoeba castellanii cysts stored at $-70{ }^{\circ} \mathrm{C}$ in $\mathrm{RM}$ with (A) $10 \%$ or $5 \%$ DMSO and (B) $80 \%$ or $40 \%$ glycerol. (A) The length of time to reach confluence increased for cysts stored with RM and 5\% DMSO the longer time spent in storage from 8.5 to 15 days whereas cysts stored with $10 \%$ DMSO varied from 8.5 to 14 days over the 12 -month period. (B) The length of time until confluence remained constant for cysts stored in $40 \%$ glycerol and RM (10-12.5 days) whereas cysts stored with $80 \%$ glycerol increased after from 11 to 24 days. The data represent the mean of 2 replicate experiments.

room temperature and then transferred to a microcentrifuge tube. Cells were collected by a 5-min centrifugation at $13000 \mathrm{rpm}$ and $4{ }^{\circ} \mathrm{C}$, resuspended in $5 \mathrm{ml}$ of $\mathrm{RM}$ the transferred to $25 \mathrm{~cm}^{2}$ flasks. All samples were monitored every $24 \mathrm{~h}$ until the flasks containing either trophozoites or cysts, which revert back to trophozoites in RM, reached $100 \%$ confluency.

\section{RESULTS}

A. castellanii trophozoites and cysts were stored under various conditions to determine their optimal long-term storage method. At 1, 3, 6 and 12 months after being placed in storage, $A$. castellanii were placed in RM until $100 \%$ confluency was reached (Figs 1-4). This demonstrated their viability. Both Acanthamoeba trophozoites and cysts remained viable in all storage media, with the exception of those stored in media containing $20 \%$ and $10 \%$ glycerol (results not shown). The length of time to reach $100 \%$ confluency increased for $A$. castellanii trophozoites stored in RM with $10 \%$ or $5 \%$ DMSO at $-70{ }^{\circ} \mathrm{C}$ from $6 \cdot 5$ to 16 days as the length of time to storage increased (Fig. 1). The time to $100 \%$ confluency also increased for $A$. castellanii cysts stored in EM at $4{ }^{\circ} \mathrm{C}$ from $8 \cdot 5$ to 24 days as the time in storage increased from 1 to 12 months (Fig. 2).

The length of time to $100 \%$ confluency increased for A. castellanii cysts stored in RM and $5 \%$ DMSO with length of time spent in storage (from 8.5 to 15 days). However, at a DMSO concentration of $10 \%$ the length of time to confluence varied throughout the entire study, with an increase from 1 month to 3 months $(8 \cdot 5$ to $12 \cdot 5$ days), then decreasing after 6 months $(8 \cdot 5$ days $)$ and finally increasing after 12 months (14 days) (Fig. 3). For cysts stored in $40 \%$ glycerol and RM the length of time to confluence remained constant (10-12.5 days), whereas for cysts stored in $80 \%$ glycerol the time required to reach trophozoite confluence increased (from 11 to 24) over the 12-month study period (Fig. 3).

The average time until confluence for A. castellanii cysts stored at $-70{ }^{\circ} \mathrm{C}$ in $\mathrm{EM}$ and in $5 \%$ or $10 \%$ DMSO varied from 9 to 13 days over the 12 -month period (Fig. 4). However, for $A$. castellanii cysts stored in EM with $80 \%$ glycerol the time to confluence increased over the study period from 13 to 24 days and cysts stored in $40 \%$ glycerol remained constant at $10-12 \cdot 5$ days (Fig. 4 ).

\section{DISCUSSION}

Cryopreservation of Acanthamoeba species has been previously described in a number of studies (Seo et al. 1992; John et al. 1994; John and John, 1996; Alejandre-Aguilar et al. 1998; Gonzalez-Robles et al. 2001). A solution containing $10 \%$ DMSO had previously been shown to produce the highest number of viable $A$. castellanii trophozoites after storage in liquid nitrogen for up to 210 days (Alejandre-Aguilar et al. 1998). Other studies have also demonstrated 

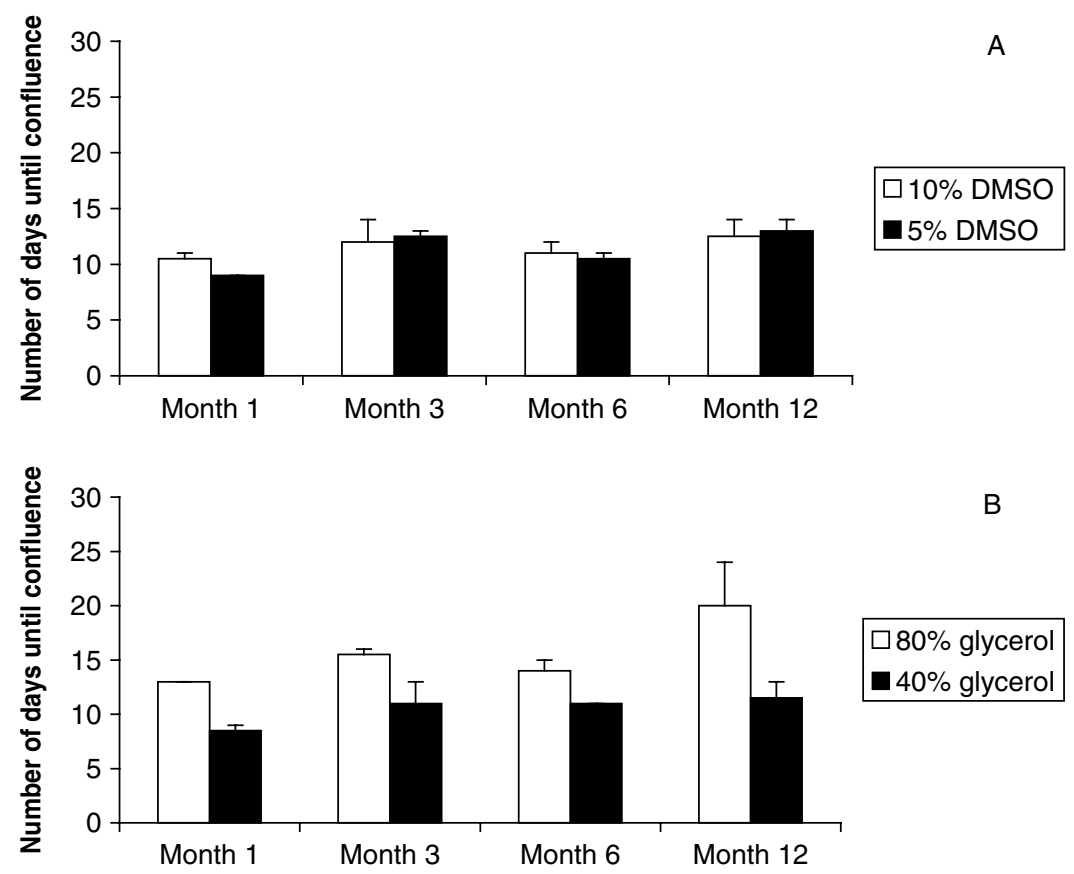

Fig. 4. The average time (days) until confluence for Acanthamoeba castellanii cysts stored at $-70{ }^{\circ} \mathrm{C}$ in EM with (A) $10 \%$ or $5 \%$ DMSO and (B) $80 \%$ or $40 \%$ glycerol. (A) The length of time for cysts in $5 \%$ or $10 \%$ DMSO varied from 9 to 13 days over the 12 -month period. (B) Cysts stored in EM with $80 \%$ glycerol took a lot longer to reach confluence (13-24 days) than cysts stored in $40 \%$ glycerol (10-12.5 days). The data represent the mean of 2 replicate experiments.

that the viability of $A$. castellanii trophozoites decreases during the first year of storage in the presence of $12 \%$ DMSO at $-70{ }^{\circ} \mathrm{C}$ (John et al. 1994). The studies undertaken herein, to investigate alternative means of storage, were instigated by similar anecdotal observations in our laboratory.

Acanthamoeba transform into cystic stages under adverse conditions and are resistant to biocides, chlorination and antibiotics. Cysts have been shown to give rise to viable trophozoites after 24 years in storage at $4{ }^{\circ} \mathrm{C}$ (Mazur et al. 1995 ; Marciano-Cabral and Cabral, 2003), but their viability has not previously been assessed relative to cryopreserved trophozoites. The results of the present study confirm that viability as determined by time to confluence is reduced in stabilates stored with DMSO or glycerol as cryopreservant following only 1 month of storage at $-70^{\circ} \mathrm{C}$. However, cysts in EM stored at $4{ }^{\circ} \mathrm{C}$ remain viable for in excess of 1 year although time to reach confluence is increased with time in storage, up to 12 months. Specifically, time to reach confluence increased from $8 \cdot 5$ to 24 days over this time-period. Viability could be markedly improved by storage at $-70{ }^{\circ} \mathrm{C}$ in the presence of either DMSO or glycerol as cryopreservant. Notably, those stored in $40 \%$ glycerol remained relatively constant throughout the whole time-period, reaching confluence at $8 \cdot 5$ days after the first month of storage and between 10 and $12 \cdot 5$ days for time-periods up to and including 1 year. Similar results were obtained using $5 \%$ and $10 \%$ DMSO as cryopreservant. The presence of $\mathrm{Mg}^{2+}$ used to induce encystment was not necessary in the cryo-medium, but did not have an adverse affect on amoeba viability.

Overall these studies demonstrate that $A$. castellanii are best stored as cyst stages rather than as trophozoites. In this state they can be easily and inexpensively preserved for at least 12 months without the need for cryopreservation. However, preservation can be improved when stored $-70{ }^{\circ} \mathrm{C}$ with cryo-preservant and this refinement is recommended for long-term storage where facilities exist.

\section{REFERENCES}

Alejandre-Aguilar, R., Calvo-Mendez, L., Nogueda-Torres, B. and de la Jara-Alcocer, F. (1998). Maintenance of Acanthamoeba culbertsoni by cryopreservation. The Fournal of Parasitology 84, 1261-1263.

Gonzalez-Robles, A., Flores-Langarica, A., Omana-Molina, M. and Shibayama, M. (2001). Acanthamoeba castellanii: ultrastructure of trophozoites using fast free fixation. Fournal of Electron Microscopy $\mathbf{5 0}$, 423-427.

Henriquez, F. L., Ingram, P. R., Muench, S. P., Rice, D. W. and Roberts, C. W. (2008). Molecular basis for resistance of Acanthamoeba tubulins to all major classes of antitubulin compounds. Antimicrobial Agents and Chemotherapy 52, 1133-1135.

John, D. T., Eddy, P. L. and John, R. A. (1994). Cryopreservation of pathogenic free-living amebae. Folia Parasitologica 41, 110-114.

John, D. T. and John, R. A. (1996). Viability of pathogenic Acanthamoeba and Naegleria and virulence 
of $N$. fowleri during long-term cryopreservation. Folia Parasitologica 43, 43-46.

Marciano-Cabral, F. and Cabral, G. (2003).

Acanthamoeba spp. as agents of disease in humans. Clinical Microbiology Reviews 16, 273-307.

Mattana, A., Biancu, G., Alberti, L., Accardo, A., Delogu, G., Fiori P. L. and Cappuccinelli, P. (2004). In vitro evaluation of the effectiveness of the macrolide rokitamycin and chlorpromazine against Acanthamoeba castellanii. Antimicrobial Agents and Chemotherapy 48, 4520-4527.

Mazur, T., Hadaś, E. and Iwanicka, I. (1995). The duration of the cyst stage and the viability and virulence of Acanthamoeba isolates. Tropical Medicine and Parasitology 46, 106-108.

McBride, J., Ingram, P. I., Henriquez, F. L. and Roberts, C. W. (2005). Development of a colourimetric microtitre plate assay for assessment of anti-microbial agents against Acanthamoeba. Fournal of Clinical Microbiology 43, 629-634.

McBride, J., Mullen, A. B., Carter, K. C. and Roberts, C. W. (2007). Differential cytotoxicity of phospholipid analogues to pathogenic Acanthamoeba species and mammalian cells. Fournal of Antimicrobial Chemotherapy 60, 521-525.

Neff, R. J., Ray, S. A., Benton, W. F. and Wilborn, M. (1964). Induction of synchronous encystment (differentiation) in Acanthamoeba sp, Methods in Cell Physiology 1, 55-83.

Schuster, F. L. (2002). Cultivation of pathogenic and opportunistic free-living amebas. Clinical Microbiology Reviews 15, 342-354.

Schuster, F. L. and Visvesvara, G. S. (2004) Free living amoebae as opportunistic and non-opportunistic pathogens of humans and animals. International fournal for Parasitology 34, 1001-1027.

Schuster, F. L. and Visvesvara, G. S. (1998). Efficacy of novel antimicrobials against clinical isolates of opportunistic amebas. Fournal of Eukaryotic Microbiology 45, 612-618.

Seo, S. A., Yong, T. S. and Im, K. (1992).

The maintenance of free-living amoebae by cryopreservation. The Korean Fournal of Parasitology 30, 151-153. 